\title{
The Tully-Fisher Relation in Numerical Simulations of Structure Formation
}

\author{
María E. De Rossi ${ }^{1,2}$, Patricia B. Tissera ${ }^{1,2}$ \& Susana E. Pedrosa ${ }^{1,2}$ \\ ${ }^{1}$ Instituto de Astronomía y Física del Espacio, CONICET-UBA, \\ CC67 Suc28, Ciudad Autónoma de Buenos Aires (1428), Argentina \\ email: derossi@iafe.uba.ar \\ ${ }^{2}$ Consejo Nacional de Investigaciones Científicas y Técnicas, \\ Rivadavia 1917, Ciudad Autónoma de Buenos Aires (1428), Argentina
}

\begin{abstract}
The Tully-Fisher Relation (TFR) is of fundamental importance for galaxy formation as it provides information about the relation between the baryonic content of galaxies and the depth of their dark halos potential wells. In recent years, it has been possible to study this relation at different redshifts. However, there are still controversies about its origin and evolution. In this work, we try to address the origin of the Tully-Fisher Relation by employing cosmological hydrodynamical simulations.
\end{abstract}

Keywords. Galaxies: formation, Galaxies: evolution, Cosmology: dark matter

\section{Introduction}

The Tully-Fisher Relation (Tully \& Fisher 1977) could provide important clues for the understanding of the dynamical evolution of galaxies as it links fundamental properties of galactic systems: their stellar or baryonic mass and total potential well. In the last decade, the large progress in observational techniques allowed the exploration of the TFR at different cosmic times (e.g. Cresci et al. 2009). However, the origin and evolution of this correlation is still not completely understood because of observational bias and systematic errors among other things. In this work, we try to address the origin of the TFR by studying cosmological hidrodynamical simulations in a hierarchical Universe. In particular, we study the influence of galactic winds on the determination of its slope and zero point.

\section{Simulations and galaxy sample}

We performed numerical simulations consistent with the concordance $\Lambda$-CDM universe with $\Omega_{\mathrm{m}}=0.3, \Omega_{\Lambda}=0.7, \Omega_{\mathrm{b}}=0.04$ and $\mathrm{H}_{0}=100 h^{-1} \mathrm{~km} \mathrm{~s}^{-1} \mathrm{Mpc}^{-1}$ with $h=0.7$. A version of GADGET-3 including treatments for metal-dependent radiative cooling, stochastic star formation, chemical enrichment and Supernovae (SN) feedback (Scannapieco et al. 2005, 2006) was employed. We adopted a Salpeter Initial Mass Function with lower and upper mass cut-off of $0.1 M_{\odot}$ and $40 M_{\odot}$, respectively. The simulated volume corresponds to a cubic box of a comoving $10 \mathrm{Mpc}^{-1}$ side length. The simulation has a mass resolution of $5.93 \times 10^{6} M_{\odot} h^{-1}$ and $9.12 \times 10^{5} M_{\odot} h^{-1}$ for the dark and gas phase respectively.

Galactic systems were identified by combining a friends-of-friends technique with the algorithm SUBFIND (Springel et al. 2001). The mean properties of galaxies were estimated at the optical radius $\left(R_{\text {opt }}\right)$, defined as the one which encloses 83 per cent of the baryonic mass of the systems. 


\section{Results and Discussion}

We studied the correlation between stellar mass and circular velocity by comparing a model which includes treatment for SN winds with another one in which this process had been suppressed. We found that both models are able to describe the linear behaviour of the observed TFR and its normalization. However, SN feedback is fundamental to reproduce the observed slope. With respect to the redshift evolution, we found that up to $z=2$ the wind-free model predicts variations smaller than 0.4 in the value of the slope. In the case of the SN-feedback model, the slope evolution is smaller than 0.1 , which is of the order of the standard deviations. This finding is important as many authors assume a constant slope when studying the evolution of the TFR. On the other hand, the zero point evolves in such a way that, at a given rotation velocity, systems become more massive with time. However, it is worth noting that we obtained a stronger evolution in the case of wind-free simulations.

We constructed also the rotation curves of simulated disk-like galaxies and estimated the rotational velocity $V_{\text {rot }}$ of the gas-phase at $R_{\text {opt }}$. With respect to the slope of the Local relation, different values have been reported in the literature but most of them ranges from 2.8 (e.g. Puech et al. 2008) to 4.5 (e.g. Bell \& de Jong 2001), depending on the selected kinematic indicator and the technique used for estimating stellar masses. Our findings are consistent with these results.

When studying the normalization of the TFR, we consider that it is more robust to employ the parameter Y100 (the TFR normalization at $V_{\text {rot }}=100 \mathrm{~km} \mathrm{~s}^{-1}$ ) rather than the zero point as the value Y100 is more representative of the data in the velocity range studied. The simulated TFR exhibit an evolution of Y100 with time in such a way that, at a given velocity, stellar masses increase by 0.2 dex in both intervals $0<z<1$ and $1<z<2$, respectively. The evolution observed between $\mathrm{z}=0$ and $\mathrm{z}=2$ is in agreement with the results of Cresci et al. (2009). At intermediate redshifts, Puech et al. (2008) found a stronger evolution, with variations of approximately $0.36 \operatorname{dex}$ between $\mathrm{z}=0$ and $\mathrm{z}=1$. However, this difference might be a consequence of the use of a different kinematic estimator and will be analized in more detail in a future work (De Rossi et al. 2009, in preparation).

\section{Acknowledgments}

We are grateful to CONICET, PICT 32342 (2005) and PICT 245 (2006). The authors would like to thank the IAU for its financial support to attend this meeting.

\section{References}

Bell, E. F. \& de Jong, R. S. 2001, ApJ, 550, 212

Cresci, G., et al. 2009, ApJ, 697, 11

Puech, M., et al. 2008, A\& A, 484, 173

Scannapieco, C., Tissera, P. B., White, S. D. M., \& Springel, V. 2006, MNRAS, 364, 552

Scannapieco, C., Tissera, P. B., White, S. D. M., \& Springel, V. 2006, MNRAS, 371, 1125

Springel, V., White, S. D. M., Tormen, G., \& Kauffmann, G. 2001, MNRAS, 328, 726

Tully, R. B. \& Fisher, R. J. 1977, A\&A, 54, 661 